\title{
Reciprocal regulation of lymphocyte activation by tyrosine kinases and phosphatases
}

\author{
Michelle L. Hermiston, ${ }^{1,2}$ Zheng Xu, ${ }^{2}$ Ravindra Majeti, ${ }^{2}$ and Arthur Weiss ${ }^{2}$ \\ ${ }^{1}$ Department of Pediatrics, and \\ ${ }^{2}$ Department of Medicine, The Howard Hughes Medical Institute, University of California, San Francisco, California, USA
}

Address correspondence to: Arthur Weiss, Department of Medicine, Box 795, 533 Parnassus Avenue, Room U-330,

University of California, San Francisco, California 94143-0795, USA.

Phone: (415) 476-1291; Fax: (415) 476-8983; E-mail: aweiss@medicine.ucsf.edu.

J. Clin. Invest. 109:9-14 (2002). DOI:10.1172/JCI200214794.

The dynamic regulation of protein tyrosine phosphorylation is crucial for a number of cellular processes including cell growth, differentiation, migration, and death. It thus represents a powerful control point for integration of environmental signals into cellular responses. Regulation of tyrosine phosphorylation is determined by the balance between protein tyrosine kinases (PTKs) and protein tyrosine phosphatases (PTPs). In the immune system, precise and coordinated regulation of this equilibrium allows for rapid responses to foreign antigens, whereas an imbalance between PTKs and PTPs can have pathologic consequences, including autoimmunity, immunodeficiency, and malignancy (1). In this review, we highlight recent advances in our understanding of key PTKs and PTPs that play critical roles in modulating cellular levels of phosphotyrosine in resting $\mathrm{T}$ cells, during $\mathrm{T}$ cell activation, and during downregulation of an immune response.

Overview of $\mathrm{T}$ cell receptor signal transduction Ligation of the $\mathrm{T}$ cell receptor (TCR) by peptide antigens presented on MHC molecules initiates a signal transduction cascade that ultimately leads to $T$ cell activation (2). The earliest event is activation of the Src family PTKs (SFKs) Lck and Fyn, which subsequently phosphorylate the immunoreceptor tyrosine-based activation motifs (ITAMs; see Billadeau and Leibson, this Perspective series, ref. 3 ) present in the $\zeta$ and $\mathrm{CD} 3 \varepsilon, \delta$, and $\gamma$ subunits of the TCR. Phosphorylated ITAMs promote recruitment and subsequent activation of ZAP-70. The activation of Src and Syk/ZAP-70 family PTKs leads to activation of the Tec family PTKs Itk and Txk. The resultant increase in PTK activity leads to phosphorylation of adapter proteins and enzymes that facilitate the stimulation of downstream signaling pathways, culminating in $\mathrm{T}$ cell activation with new gene transcription, cytoskeletal reorganization, cytokine production, and proliferation (reviewed in refs. 2, 4).

The rapidity with which the above events are initiated upon antigen encounter is consistent with the existence of an ultrasensitive switch that translates environmental information into cellular responses (5). Tyrosine phosphorylation plays a central and critical role in regulating this switch. Increased protein tyrosine phosphorylation is detectable within seconds of TCR engagement. In addition, PTK inhibitors impair T cell activation, whereas PTP inhibitors can activate resting $T$ cells, even in the absence of other stimuli $(6,7)$. Hence, the maintenance of $\mathrm{T}$ cells in a resting state is an active process involving a dynamic equilibrium between PTPs and PTKs. T cell activation represents a shift in this equilibrium.

The active segregation of components of signal transduction cascades, particularly PTPs and PTKs, represents a potentially powerful mechanism for reciprocal regulation of protein tyrosine phosphorylation. Recent studies suggest that subcellular localization of PTKs, PTPs, and their substrates changes with developmental state and with the activation status of T cells $(8,9)$. Membrane subdomains enriched in glycosphingolipids, cholesterol, and other specialized lipids have been identified and variably termed glycosphingolipid-enriched micro domains (GEMs), detergent-insoluble glycolipidrich membranes (DIGs), or lipid rafts (see Dustin, this Perspective series, ref. 10). Accumulating data indicate that certain proteins, such as linker for activated $\mathrm{T}$ cells (LAT), Ras, and Lck, are associated with rafts, whereas CD45 and ZAP-70, among others, are excluded from these domains in resting T cells (9). T cell activation results in recruitment to the raft of additional signaling components, such as the TCR itself and Syk/ZAP-70. CD45 remains persistently excluded from the rafts. At the interface of the $T$ cell and antigen-presenting cell, raft coalescence may occur during the formation of a supramolecular activation cluster, an organized contact interface also termed the immunological synapse (ref. 8; see also Dustin, this series, ref. 10).

Regulated subcellular localization will result in local alterations in enzyme and substrate concentration, which may amplify the switchlike response to environmental cues and modify the signaling threshold required for $\mathrm{T}$ cell activation. Altering the threshold at which external signals trigger the intracellular switch 


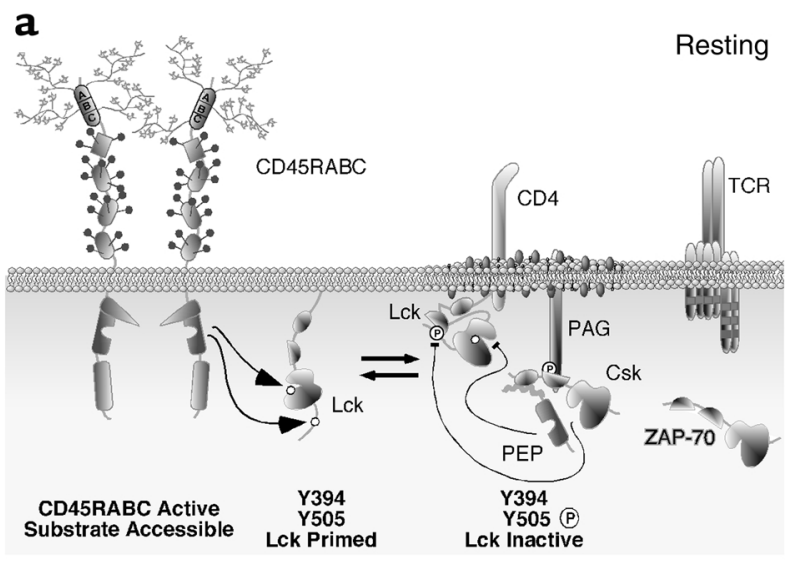

b
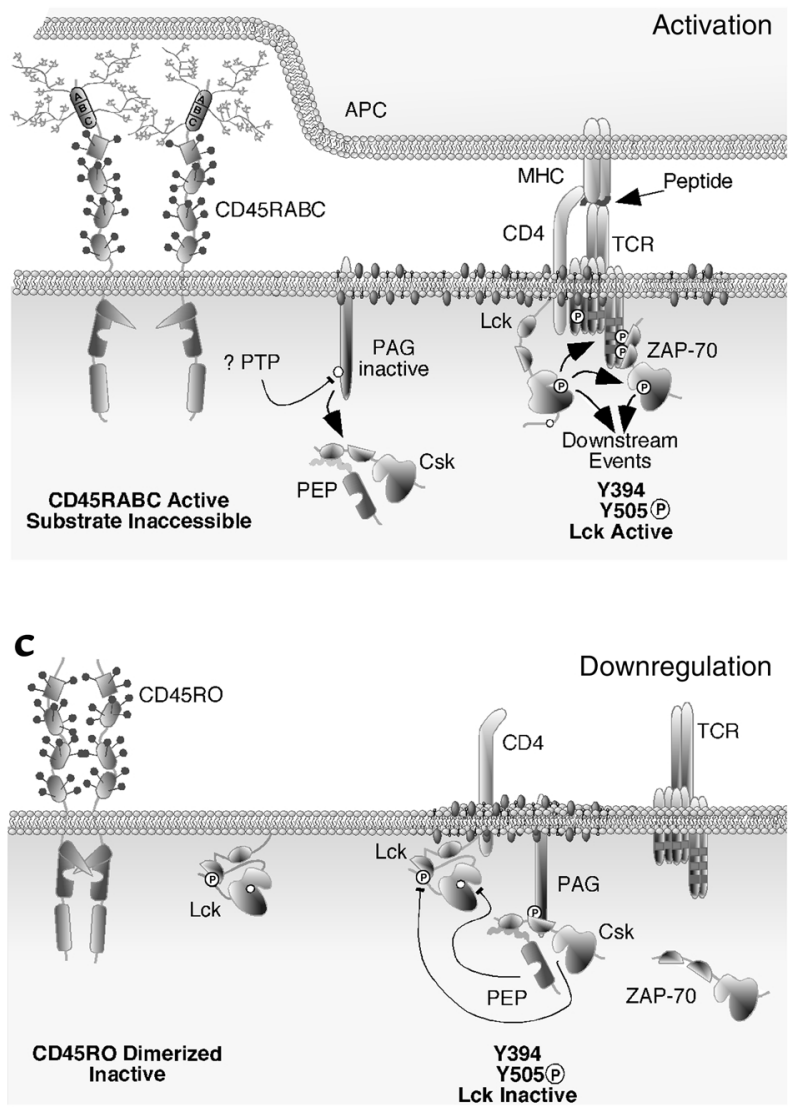

represents an economical means by which cells can use the same signal transduction machinery to produce different responses in different developmental and/or cellular contexts. For example, active regulation of phosphotyrosine levels in resting cells may be essential for maintenance of a basal level of signaling necessary to keep the cell in a "primed" state, capable of rapid response if foreign antigen appears. Raising the threshold during $\mathrm{T}$ cell downregulation may be important for limiting the $T$ cell response, allowing elimination of excess cells, and facilitating a return to homeostasis. Subsequently lowering the threshold in the surviving memory cells may enable a more rapid and robust response to reencounter of antigen. While many details remain to be elucidated, the reciprocal regulation of

\section{Figure 1}

Speculative model for reciprocal regulation of tyrosine phosphorylation in lymphocytes. We hypothesize that Lck traffics between rafts and other subcellular pools based upon its tyrosine phosphorylation status. (a) Thus, in the resting T cell, Lck is in a dynamic equilibrium between its inactive and primed conformations due to the reciprocal activity of the phosphatase CD45RA+ and the kinase Csk/PEP. (b) Upon T cell activation, rafts coalesce and an immunological synapse forms. CD45RA+ is excluded from the immunological synapse due to its size. PAG is dephosphorylated by an unknown phosphatase, resulting in release of Csk/PEP. Unopposed, primed Lck is thus able to undergo transphosphorylation at Y394, which yields an active kinase that subsequently phosphorylates the ITAMs of the CD3 and $\zeta$ chains. The phosphorylated ITAMs provide docking sites for the SH2 domains of ZAP-70. ZAP-70 is further phosphorylated by Lck, and activation of downstream signaling events ensues. (c) During downregulation, the CD45RO isoform is inactivated by homodimerization. PAG is again phosphorylated, recruiting Csk/PEP to the raft, where Lck becomes phosphorylated at Y505 and dephosphorylated at $\mathrm{Y} 394$, leaving this kinase in an inactive conformation.

PTK Lck by the PTP CD45 and the PTK Csk represents the best-characterized example of this concept and will be the focus of the remainder of this review.

\section{Dynamic regulation of SFKs} by tyrosine phosphorylation

SFKs are nonreceptor PTKs that function as molecular switches to regulate cell growth, differentiation, migration, and survival (11). Lck and Fyn are the predominant SFK members in T cells. Lck plays a special role in T cells, as it is noncovalently associated with the CD4 coreceptor. Studies in Lck-deficient cell lines and mice demonstrate its central role in $\mathrm{T}$ cell signal transduction. Lckdeficient Jurkat $\mathrm{T}$ leukemic cells are unable to initiate TCR signal transduction following receptor cross-linking, as evidenced by the lack of ITAM phosphorylation, calcium flux, or any other events associated with TCR signaling function. These responses are restored upon reintroduction of wild-type Lck (12). Lck-deficient mice have a profound block in $T$ cell development with arrest at the pre-TCR checkpoint, the first developmental stage requiring TCR signaling machinery (13).

All nine SFKs share a similar structure, including an $\mathrm{N}$-terminal unique domain, containing myristoylation and, in some cases, palmitoylation signals, that facilitate targeting to the plasma membrane; an Src homology-2 (SH2) domain, involved in associations with tyrosine phosphorylated residues; an SH3 domain, capable of interactions with proline-rich polypeptides; a catalytic domain; and a short $\mathrm{C}$-terminal negative regulatory domain (11). All SFKs contain two tyrosine phosphorylation sites that regulate kinase function. Phosphorylation of the first (corresponding in Lck to Y394), located in the kinase domain, potentiates kinase activity by repositioning a loop within the catalytic domain for optimal phosphotransferase activity. Conversely, phosphorylation of the carboxy-terminal tyrosine (Y505 in Lck) negatively regulates kinase activity. Analysis of the crystal structures of c-Src and Hck provide a molecular model for the negative regulation of catalytic activity (11). Phosphorylation of the carboxy-terminal, negative regulatory tyrosine of Src provides a binding site for its own SH2 domain, facilitating an intramolecular inter- 
action that helps maintain Src in a "closed," inactive conformation in which the substrate binding site is blocked. This closed conformation is stabilized by an interaction of the Src SH3 domain with a hinge sequence between the $\mathrm{SH} 2$ and kinase domains. Recent molecular dynamic simulations indicate that the connector region between the $\mathrm{SH} 2$ and $\mathrm{SH} 3$ domains also is crucial for stabilizing the kinase in the inactive closed conformation (14). It appears that this connector functions as a "snap lock" that couples the SH2 and SH3 domains in a configuration that impedes access to the catalytic domain. Dephosphorylation of the C-terminal tail or the binding of ligands to the $\mathrm{SH} 2$ or the $\mathrm{SH} 3$ domains restores flexibility to the connector domain and allows the kinase to adopt a more open primed conformation. Subsequent clustering of SFKs on the cell surface may allow for transphosphorylation of the catalytic domain and full activation of kinase activity.

Because of their proximal position in TCR signal transduction and rapid activation with TCR cross-linking, modulation of SFK phosphorylation status represents a critical regulatory node in T cells. This is highlighted in transgenic mice expressing an Lck mutant in which the C-terminal tyrosine Y505 is mutated to phenylalanine to prevent phosphorylation at this site (15). These mice have hyperactivated $\mathrm{T}$ cells and develop thymomas in a transgene dose-dependent manner. Biochemical analysis of Lck phosphorylation status in these transgenic $\mathrm{T}$ cells reveals that the catalytic domain tyrosine is more heavily phosphorylated, correlating with increased kinase activity. These findings suggest that the regulation of two sites of phosphorylation of SFKs and their effects on kinase function are intimately connected. As discussed in detail below, regulation of net SFK phosphorylation is a complex process requiring the opposing actions of the PTK C-terminal Src kinase (Csk), which phosphorylates the negative regulatory tyrosine, and the PTP CD45, which opposes this action (Figure 1).

\section{Role of the receptor-like PTP CD45}

\section{in regulation of immune cell function}

CD45 is a receptor- like PTP (RPTP) expressed on all nucleated hematopoietic cells. Consistent with a critical role for CD45 in T and B cell signal transduction, CD45-deficient $T$ and $B$ cell lines fail to respond to antigen receptor stimulation, and patients with CD45 deficiency have a severe combined immunodeficiency phenotype. CD45-deficient mice likewise have a profound block in $\mathrm{T}$ and $\mathrm{B}$ cell development and function (refs. 16, 17 and references therein). One key function of CD45 is to serve as a positive regulator of SFK by opposing Csk function and by dephosphorylating the negative regulatory C-terminal tyrosine of SFKs. Dephosphorylation of this site maintains SFKs in a primed, or signal-competent, state capable of full activation upon receptor stimulation. Consistent with this hypothesis, SFKs have decreased kinase activity and are hyperphosphorylated at the negative regulatory tyrosine in most CD45-deficient cells. Moreover, expression of a constitutively active Lck Y505F mutant in CD45-deficient mice largely rescues $\mathrm{T}$ cell development $(18,19)$. CD45 may also dephosphorylate the autocatalytic tyrosine in
SFKs, albeit less efficiently. Dephosphorylation of the C-terminal tyrosine leaves the SFKs in an unphosphorylated primed conformation, ready for rapid activation. As discussed below, other PTPs have also been implicated in the dephosphorylation of this site.

A recent report suggests that CD45 may also be involved in the reciprocal regulation of a second family of PTKs, the Janus kinases (JAKs) (20). JAKs function as positive regulators of cytokine-mediated signal transduction by phosphorylating the members of the signal transducer and activator of transcription (STAT) family of transcription factors. The STATs then translocate to the nucleus, where they regulate the expression of genes involved in cytokine and chemokine responses. Recent experiments employing CD45-deficient cell lines and mice suggest that CD45 acts as a negative regulator of JAKs (20). CD45-deficient mice have increased cytokinedependent myelopoiesis and erythropoiesis and corresponding hyperphosphorylation of JAK2 and STATs 3 and 5. CD45 deficiency also protects mice from the lethal cardiomyopathy that results from Coxsackie virus B3 (CVB3) infection. While intriguing, a role for CD45 as a JAK phosphatase must be viewed cautiously, since the phenotype observed could also be attributed to inhibition of SFK activity in the CD45-deficient mice. However, it is becoming increasingly clear that SFKs activate STATs, either in parallel with or downstream of JAKs (21). The observation that Lck-deficient mice are also protected from CVB3 infection provides support for the notion that some effects of CD 45 ascribed to JAK kinase effects may be mediated by its effects on SFKs (22). Additional work is needed to clarify the role of CD45 in regulation of JAK function.

Given the critical role of CD45 function in T cell biology, it is not surprising that its regulation has become an area of intense research. Current data suggest two potential mechanisms for CD45 regulation: localization and receptor dimerization. Although it is clear that CD45 plays a positive role in $\mathrm{T}$ cell activation, other studies indicate it can function as a negative regulator in other contexts (refs. 17, 18 and references therein). CD45-deficient macrophages and $T$ cells are abnormally adherent, a phenotype that is reversed with the addition of wildtype CD45 (17). This effect has been ascribed to negative regulation of SFKs by CD45 during integrin-mediated signal transduction. Surprisingly, despite the fact that the negative regulatory tyrosine of the SFKs is hyperphosphorylated in the CD45-deficient macrophages, kinase activity is actually enhanced, due to hyperphosphorylation of the autocatalytic site. Thus, the distribution of SFK phosphorylation may account for the increased adhesiveness of these cells, despite the seemingly paradoxical positive and negative regulation of SFKs by CD45. Apparently, CD45's effect depends upon its localization in relation to its substrate, such that physical separation of CD45 from the TCR during antigen recognition at the immunological synapse results in a net positive effect, whereas access to its substrate during integrin-mediated adhesion results in a negative effect. The biochemical and microscopic evidence that CD45, but not Lck, is excluded from lipid rafts and the immunological synapse $(8,9)$ is consistent with this 
model. In resting T cells, CD45 may counteract the negative regulation of Csk by dephosphorylating the negative regulatory, and to a lesser extent the autocatalytic, tyrosine in SFKs, providing a pool of signal-competent Lck. The latter kinase would then be available to phosphorylate the TCR ITAMs and bound ZAP-70 whenever foreign antigen is encountered. During antigen recognition, TCR clustering in the central region of the immunologic synapse, a process that evolves over time, would result in functional segregation of CD45 from its substrate and would sustain Lck activity during initiation of signal transduction cascades (Figure 1, a and b).

Receptor dimerization may represent a second means of regulating $\mathrm{CD} 45$ function. Like most members of the RPTP family, CD45 consists of an extracellular domain, a single transmembrane domain, and a cytoplasmic domain containing tandemly duplicated PTP domains (23). CD45 exists as multiple isoforms due to alternative splicing of exons 4,5 , and 6 , which encode a portion of the extracellular domain. The alternatively spliced exons encode multiple sites of O-linked glycosylation, which are variably modified by sialic acid. Thus, the extracellular domain of high-molecular weight isoforms $(\mathrm{CD} 45 \mathrm{RA}+)$ differs in structure and overall charge from the low-molecular weight isoform (CD45RO) lacking these exons. Isoform expression is highly regulated in a cell- and activation state-specific manner. For example, in T cells, alternative splicing of CD45 is regulated such that naive $T$ cells predominantly express CD45RA isoforms containing exons 4 and 5 or 5 and 6. Upon activation, alternative splicing machinery is induced, in a Ras-dependent manner, to favor the exclusion of exons 4,5 , and 6 , resulting in the expression of the lower-molecular weight CD45RO isoform (24). The tight control of CD45 isoform expression supports the notion that the extracellular domain could play a central role in regulation of CD45 function by facilitating isoform-specific differential interaction with a ligand and/or differential homodimerization.

A definitive ligand for CD45 has not been established. However, several lines of evidence suggest dimerization may regulate CD45 function. First, dimeric forms of CD45 have been identified $(25,26)$. Second, work with a chimeric protein consisting of the extracellular and transmembrane domains of the EGF$\mathrm{R}$ fused to the cytoplasmic domain of CD45 (27) suggests that dimerization directly suppresses CD 45 activity. This chimera is sufficient to restore TCR-mediated signal transduction in a CD45-deficient $\mathrm{T}$ cell line, but its activity is lost when the EGF is provided to induce dimerization. Hence, dimerization of the native CD45 - either induced by some as-yet unidentified ligand or occurring spontaneously - would be expected to silence TCR-mediated signal transduction.

A molecular explanation for this inhibition was provided by the crystal structure of the juxtamembrane and proximal catalytic domain of a related phosphatase, RPTP $\alpha$ (28). This protein fragment forms a symmetrical dimer in which the catalytic site of one molecule is blocked by specific contacts with a wedge formed by the juxtamembrane region of the dimer partner. The dimeric PTP is thus inhibited by mutual occlusion of the active sites. The homologous region within the juxtamembrane domain of CD45 is remarkably conserved phylogenetically, suggesting a preserved evolutionary function. This region of CD45 could be modeled into a wedge-like structure, based on the RPTP $\alpha$ structure. Mutation of a highly conserved acidic amino acid that is predicted to reside at the tip of the CD45 wedge domain significantly blunts the inhibitory effect of dimerization in the experiments using the EGF-R-CD45 chimera (29).

We recently tested the physiologic significance of this model by generating an analogous mutation (E613R) in vivo by homologous recombination (26). In mice carrying the CD45 E613R mutation, $\mathrm{T}$ and $\mathrm{B}$ cell development is initially normal, but at $12-16$ weeks of age, homozygous mutant mice develop a lymphoproliferative disorder with polyclonal expansion of activated $\mathrm{T}$ and $\mathrm{B}$ cells, autoantibody production, and severe autoimmune nephritis. These animals die prematurely, and their phenotype is consistent with hyperactivity of SFK-mediated pathways and suggests that dimerization plays a critical negative regulatory role in $\mathrm{CD} 45$ function in vivo.

The identification of dimeric forms of CD45 and RPTP $\alpha$ in the absence of exogenously added protein suggests that spontaneous homodimerization occurs. This finding has led us to question the need for a putative endogenous CD45 ligand that might drive dimerization of this phosphatase. We hypothesize that differential isoform expression may regulate $\mathrm{CD} 45$ homodimerization and function. The extensive O-linked glycosylation and sialylation of the alternatively spliced exons in CD45RA+ impart on the protein a strong negative charge that may form an electrostatic repulsive barrier to homodimerization. The lack of these modifications in the CD45RO isoform may allow more efficient homodimerization. We propose that the CD45RA+-to-CD45RO switch would allow for more efficient dimer formation, downregulating CD45 PTP activity and contributing to the cessation of the primary $\mathrm{T}$ cell response (Figure 1c). The wedge mutation in the E613R mice eliminates the negative regulatory effect of CD45RO homodimerization, resulting in persistent $\mathrm{T}$ cell activation and autoimmune disease.

Genetic support for the importance of the RA-to-RO isoform switch during the primary immune response comes from identification of a single nucleotide polymorphism, silent at the protein level, in exon 4 of the human $C D 45$ gene. This alteration prevents the switch from RA- to RO-encoding splicing forms. According to the above model, individuals with this mutation would be unable to regulate $\mathrm{CD} 45$ by dimerization and could be predisposed to autoimmune disease. Consistent with this hypothesis, population studies have recently demonstrated that this mutation is associated in a small subset of German individuals with the development of multiple sclerosis (30). While intriguing, a causal role for this mutation in disease development awaits further analysis.

\section{Negative regulation by Csk and the associated proteins PEP and Cbp/PAG}

Csk is a ubiquitously expressed cytosolic PTK. As with SFKs, Csk contains an amino-terminal SH3 domain, an $\mathrm{SH} 2$ domain, and a catalytic kinase domain. However, Csk plays a negative regulatory role in cells by inhibit- 
ing intracellular processes induced by SFKs. Gene targeting experiments show that Csk is essential for development, since animals lacking Csk have dramatically elevated levels of c-Src activity and die at embryonic day $9(31,32)$. Csk also plays a key role in negatively regulating TCR-mediated signal transduction. Overexpression of Csk resulted in a decrease in total cellular phosphotyrosine in response to TCR cross-linking and a corresponding decrease in IL-2 production (33). A conditional gene targeting approach shows that Csk plays a critical role in setting the threshold for antigen receptor signal transduction in thymocytes. Inactivation of Csk disrupts the pre-TCR developmental checkpoint that permits TCR $\alpha \beta$ - and MHC class II-independent development of $\mathrm{CD}^{+} \mathrm{T}$ cells (34). These analyses suggest a differential role of Csk-mediated regulation of SFKs during thymopoiesis, since positive selection of Csk-deficient thymocytes, but not negative selection, can be uncoupled from TCR-derived input.

Structure-function studies have demonstrated that the $\mathrm{SH} 2, \mathrm{SH} 3$, and catalytic domains are all required for Csk to function as a negative regulator. The SH3 domain of Csk constitutively binds with high affinity to a family of cytoplasmic PTPs, including the hematopoietic cell-specific PEP and the ubiquitously expressed PTP-PEST $(35,36)$. Interestingly, PEP family PTPs dephosphorylate the positive regulatory tyrosine of SFKs. When in a complex together, the functional effects of both the kinase Csk and the phosphatases PEP and PTP-PEST are augmented, suggesting that these enzymes act synergistically to inactive SFKs and to suppress TCR signaling (37). A third PEP family member, PTP-HSCF, has recently been described. Unlike PEP and PTP-PEST, the interaction between Csk and PTP-HSCF involves the Csk SH2 domain and conserved tyrosines in the $\mathrm{C}$-terminal noncatalytic region of PTP-HSCF (38). SFK-dependent phosphorylation of these tyrosines enhances PTP-HSCK/Csk binding and facilitated the capacity of each to inhibit SFKs. This is consistent with a model in which SFKs activate a negative feedback loop to downregulate their own activity.

The relative contribution of the different PEP family PTPs in regulating SFK function remains to be elucidated. While they may simply be redundant, the observations that they accumulate in distinct intracellular compartments and that their interactions with Csk can be induced independently, as in the case of PTP-HSCF, raises the possibility that these molecules may have distinct functions aimed at inhibiting separate intracellular pools of SFKs.

Adding to the complexity, an additional Csk family, Chk, has been described (39). Its function remains to be elucidated. Unlike Csk, Chk is selectively expressed in hematopoietic cells and the brain and is not capable of interacting with PEP family PTPs. This could explain its relative inefficiency at negatively regulating antigen receptor signaling in T cells. An initially perplexing feature of Csk regulation of SFKs was the differential localization of these proteins within the cell. Unlike SFKs that are anchored to the inner leaflet of the plasma membrane through $\mathrm{N}$-terminal lipid modifications, Csk is a cytosolic protein and has no sites for lipid modification. Hence, one means of dynamically regulating Csk function would be by controlling its subcellular localization, as several recent reports suggest. The Csk SH2 domain interacts specifically with several tyrosine-phosphorylated molecules, including Dok family molecules, paxillin, tensin, Fak, and the

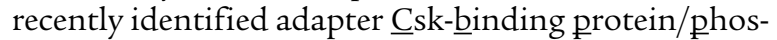
phoprotein associated with glycosphingolipid-enriched microdomains (Cbp/PAG) (9). These interactions may facilitate targeting of Csk to sites of SFK activation.

The significance of such interactions has been most clearly established for $\mathrm{Cb} / \mathrm{PAG}$, a ubiquitously expressed type III transmembrane protein similar in structure to the well-known adapter, LAT (40-42). Cbp/PAG contains a short amino-terminal extracellular domain, a transmembrane region, a cytoplasmic tail containing a palmitoylation motif, two potential SH3 binding motifs, and ten potential tyrosine phosphorylation sites. Like SFKs and LAT, $\mathrm{Cb}$ /PAG has been shown to be palmitoylated and targeted to rafts. In resting human $\mathrm{T}$ cells, $\mathrm{Cb} / \mathrm{PAG}$ is constitutively phosphorylated at Y314 by SFKs (41). Phosphorylation at this site results in recruitment of Csk to the GEM. Analogous to PEP binding, Cbp/PAG-Csk interaction increases Csk catalytic activity on its substrate, thereby inhibiting SFK function. Interestingly, the $\mathrm{Cbp} / \mathrm{PAG}-\mathrm{Csk}$ interaction is reversible. In one recent study, the activation of peripheral blood $\mathrm{T}$ cells resulted in rapid dephosphorylation of $\mathrm{Cb} / \mathrm{PAG}$ and caused the release of Csk (41). Hence, one function of $\mathrm{Cbp} / \mathrm{PAG}$ may be to target Csk to the membrane, increasing the signal threshold required for initiating an immune response, and thereby actively maintaining lymphocytes in a resting state in the absence of external stimuli (Figure 1a). Upon antigen receptor stimulation, the dephosphorylation of $\mathrm{Cbp} / \mathrm{PAG}$ results in the release of the Csk/PEP complex, which promotes the activation of SFKs participating in the response (Figure $1 \mathrm{~b}$ ).

Several important issues remain. A recent study using biochemical and confocal microscopy techniques to determine subcellular localization of PTP in T cells found that, whereas the majority of PEP is cytoplasmic, a small fraction occurs in membrane and nuclear fractions (43). It will be important to determine whether Csk and/or Cbp/PAG are complexed with PEP in these sites. The timing and mechanism of Cbp/PAG dephosphorylation also needs to be elucidated, as does its phosphorylation state and its interaction with Csk during the downregulation of immune responses. Segregation of Csk from SFKs during initial lymphocyte activation is obviously crucial, but it is not yet clear how the complex forms again after this step. The regulated localization of Csk and its partners may play an equally important role in terminating an immune response. Microscopic visualization of protein localization in real time, as well as by genetically engineered manipulation of $\mathrm{Cbp} / \mathrm{PAG}$ and PEP family member expression, should help clarify these matters. In particular, although simple knockout of these proteins may be embryonic-lethal, reflecting their ubiquitous expression, tissue-specific knockouts or subtle knock-in mutations in Cbp/PAG to remove regulatory phosphorylation sites should be enlightening. We predict that eliminating expression early in thymopoiesis 
will allow TCR-independent development, similar to the tissue-specific knockout of Csk. Eliminating expression in peripheral $\mathrm{T}$ cells could result in autoimmunity due to unopposed SFK activity, similar to the CD45 wedgemutant mice, or more likely in lymphomas as is the case for Lck Y505F mutants.

\section{Conclusions}

While it is clear that the regulation of tyrosine phosphorylation and associated signaling through antigen receptors is mediated by the reciprocal activities of PTKs and PTPs, many issues remain. The relative importance of CD45 and of the Csk/Cbp/PAG/PEP complex in the regulation of the phosphotyrosine status of SFKs has yet to be addressed. Current data support a model in which these enzymes function in a dynamic equilibrium to maintain resting cells in a primed state. Regulated subcellular localization, allowing for the amplification of functional activities by segregating kinases from phosphatases, is likely to be critical for T cell activation. The biochemical approaches used to date have been limited by the fact that they measure total cellular pools of protein. New approaches are needed that allow assessment of phosphorylation status and kinase activity of subcellular pools, and, ultimately, of individual molecules in real time. Furthering our understanding in this area will help explain the pathogenesis of autoimmune disease and will likely suggest novel approaches for rational therapeutic design.

\section{Acknowledgments}

We thank members of the Weiss lab for valuble discussion and J. Lin for assistance with the figure. This work was supported in part by NIH ROIGM 395, by the Medical Scientist Training Program, and by the Howard Hughes Medical Institute. We apologize to colleagues for not citing many pertinent works due to space limitations.

1. Li, L., and Dixon, J.E. 2000. Form, function, and regulation of protein tyrosine phosphatases and their involvement in human diseases. Semin. Immunol. 12:75-84.

2. Kane, L.P., Lin, J., and Weiss, A. 2000. Signal transduction by the TCR for antigen. Curr. Opin. Immunol. 12:242-249.

3. Billadeau, D.D., and Leibson, P.J. 2002. ITAMs versus ITIMs: striking a balance during cell regulation. J. Clin. Invest. In press.

4. Tomlinson, M.G., Lin, J. and Weiss, A. 2000. Lymphocytes with a complex: adapter proteins in antigen receptor signaling. Immunol. Today. 21:584-591.

5. Ferrell,J.E. 1998. How regulated protein translocation can produce switchlike responses. Trends Biochem. Sci. 18:416-465.

6. Hsi, E.D., et al. 1989. T cell activation induces rapid tyrosine phosphorylation of a limitednumber of cellular substrates. J. Biol. Chem. 264:10836-10842.

7. Seclist, J.P., Burns, C.A., Karnitz, L. Koretsky, G.A., and Abraham, R.T. 1993. Stimulatory effects of protein tyrosine phosphatse inhibitor, pervanadate, on T cell activation events. J. Biol. Chem. 268:5888-5893.

8. van der Merwe, P.A., Davis, S.J., Shaw, A.S., and Dustin, M.L. 2000 Cytoskeletal polarization and redistribution of cell-surface molecules during $\mathrm{T}$ cell antigen recognition. Semin. Immunol. 12:5-21.

9. Janes, P.W., Ley, S.C., Magee, A.I., and Kabouridis, P.S. 2000. The role of lipid rafts in T cell antigen receptor (TCR) signalling. Semin. Immunol. 12:23-34.

10. Dustin, M. 2002. Membrane domains and the immunological synapse: keeping T cells resting and ready. J. Clin. Invest. In press.

11. Bjorge, J.D., Jakymiw, A., and Fujita, D.J. 2000. Selected glimpses into the activation and function of Src kinase. Oncogene. 19:5620-5635.

12. Straus, D., and Weiss, A. 1992. Genetic evidence for the involvement of the Lck tyrosine kinase in signal trasudction through the T cell antigen receptor. Cell. 70:585-593.

13. Molina, T.J., et al. 1992. Profound block in thymocyte development in mice lacking p56lck. Nature. 357:161-164.

14. Young, M.A., et al. 2001. Dynamic coupling between the SH2 and SH3 domains of c-Src and Hck underlies their inactivation by C-terminal tyrosine phosphorylation. Cell. 105:115-126.

15. Abraham, K.M., Levin, S.D., Marth, J.D., Forbush, K.A., and Perlmutter, R.M. 1991. Thymic tumorigenesis induced by overexpression of p56Lck. Proc. Natl. Acad. Sci. USA. 88:3977-3981.

16. Thomas, M.L. and Brown, E.J. 1999. Positive and negative regulation of Srcfamily membrane kinases by CD45. Immunol. Today. 20:406-411.

17. Alexander, D.R. 2000. The CD45 tyrosine phosphatase: a positive and negative regulator of immune cell function. Semin. Immunol. 12:349-359.

18. Pingel, S., Baker, M., Turner, M., Holmes, N., and Alexander, D.R. 1999. The CD45 tyrosine phosphatase regulates CD3-induced signal transduction and $T$ cell development in recombinase-deficient mice: restoration of preTCR function by active p56lc. Eur. J. Immunol. 29:2376-2384.

19. Seavitt, J.R., et al. 1999. Expression of the p56 ${ }^{\text {lck }}$ Y505F mutation in CD45deficient mice rescues thymocyte development. Mol. Cell. Biol. 19:4200-4208.

20. Irie-Sasaki, J., et al. 2001. CD45 is a JAK phosphatase and negatively regulates cytokine receptor signalling. Nature. 409:349-354.

21. Reddy, E.P., Korapati, A., Chaturvedi, P., and Rane, S. 2000. IL-3 signaling and the role of Src kinases, JAKs and STATs: a covert liaison unveiled. Oncogene. 19:2532-2547.

22. Liu, P., et al. 2000. The tyrosine kinase p56Lck is essential for coxsackievirus B3-mediated heart disease. Nat. Med. 6:429-434.

23. Majeti, R., and Weiss, A. 2001. Regulatory mechanisms for receptor protein tyrosine phosphatases. Chem. Rev. 101:2441-2448.

24. Lynch, K.W., and Weiss, A. 2000. A model system for activation-induced alternative splicing of CD45 pre-mRNA in T cells implicates PKC and Ras. Mol. Cell. Biol. 20:70-80.

25. Takeda, A., Wu, J.J., and Maizel, A.L. 1992. Evidence for monomeric and dimeric forms of CD45 associated with a $30 \mathrm{kDa}$ phosphorated protein. J. Biol. Chem. 267:16651-16659.

26. Majeti, R., et al. 2000. An inactivating point mutation in the inhibitory wedge of CD45 causes lymphoproliferation and autoimmunity. Cell. 103:1059-1070.

27. Desai, D.M., Sap, J., Schlessinger, J., and Weiss, A. 1993. Ligand-mediated negative regulation of a chimeric transmembrane recetpor tyrosine phosphatase. Cell. 73:541-554.

28. Jiang, G., et al. 1999. Dimerization inhibits the activity of receptor-like protein tyrosine phosphatase-alpha. Nature. 401:606-610.

29. Majeti, R., Bilwes, N.J.P., Hunter, T., and Weiss, A. 1998. Ligand-induced dimerization regulates receptor protein tyrosine phosphatase function via an inhibitory wedge. Science. 279:88-91.

30. Jacobsen, M., et al. 2000. A point mutation in PTPRC is associated with the development of multiple sclerosis. Nat. Genet. 26:495-499.

31. Nada, S., et al. 1993. Constitutive activation of Src family kinases in mouse embryos that lack Csk. Cell. 73:1125-1135.

32. Imamoto, A., and Soriano, P. 1993. Disruption of the csk gene, encoding a negative regulator of Src family tyrosine kinases, leads to neural tube defects and embryonic lethality in mice. Cell. 73:1117-1124.

33. Chow, L.M.L., Fournel, M., Davidson, D., and Veillette, A. 1993. Negative regulation of T-cell receptor signalling by tyrosine protein kinase $\mathrm{p} 50^{\mathrm{cks}}$. Nature. 365:156-159.

34. Schmedt, C., and Tarakhovsky, A. 2001. Autonomous maturation of $\alpha / \beta$ T lineage cells in the absence of COOH-terminal Src kinase (Csk). J. Exp. Med. 193:815-825.

35. Cloutier, J.-F., and Veillette, A. 1996. Association of inhibitory tyrosine protein kinase $\mathrm{p} 50 \mathrm{csk}$ with protein tyrosine phosphatase PEP in T cells and other hemopoietic cells. EMBOJ. 15:4909-4918.

36. Davidson, D., Cloutier, J.-F., Gregorieff, A., and Veillette, A. 1997. Inhibitory tyrosine protein kinase $\mathrm{p} 50^{\text {csk }}$ is associated with protein-tyrosine phosphatase PTP-PEST in hemopoietic and non-hemopoietic cells. J. Biol. Chem. 272:23455-23462.

37. Cloutier, J.-F., and Veillette, A. 1999. Cooperative inhibition of T-cell antigen receptor signaling by a complex between a kinase and a phosphatase. J. Exp. Med. 189:111-121.

38. Wang, B., Lemay, S., Tsai, S., and Veillette, A. 2001. SH2 domain-mediated interaction of inhibitory protein tyrosine kianse Csk with protein tyrosine phosphatase-HCSF. Mol. Cell. Biol. 21:1077-1088.

39. Davidson, D., Chow, L.M., and Veillette, A. 1997. Chk, a Csk family tyrosine protein kinase, exhibits Csk-like activity in fibroblasts but not in an antigen-specific T cell line. J. Biol. Chem. 272:1355-1362.

40. Kawabuchi, M., et al. 2000. Transmembrane phosphoprotein Cbp regulates the activities of Src-family tyrosine kinases. Nature. 404:999-1003.

41. Brdicka, T., et al. 2000. Phosphoprotein associated with glycosphingolipidenriched microdomains (PAG), a novel ubiquitously expressed transmembrane adaptor protein, binds the protein tyrosine kinase Csk and is involved in regulation of T cell activation. J. Exp. Med. 191:1591-1604.

42. Takeuchi, S., Takayama, Y., Ogawa, A., Tamura, K., and Okada, M. 2000. Transmembrane phosphoprotein $\mathrm{Cbp}$ positively regulates the activity of the carboxyl-terminal kinase, Csk. J. Biol. Chem. 275:29183-29186.

43. Gjorloff-Wingren, A., et.al. 2000. Subcellular localization of intracellular protein tyrosine phosphatases in T cells. Eur. J. Immunol. 30:2412-2421. 